\title{
Impact écotoxicologique du traitement chimique des eaux usées de tanneries: Analyse technico-économique Impact écotoxicologique du traitement chimique des eaux usées de tanneries: Analyse technico-économique
}

\author{
A. Yatribi et A. Nejmeddine
}

Volume 13, numéro 2, 2000

URI : https://id.erudit.org/iderudit/705384ar

DOI : https://doi.org/10.7202/705384ar

Aller au sommaire du numéro

\section{Éditeur(s)}

Université du Québec - INRS-Eau, Terre et Environnement (INRS-ETE)

\section{ISSN}

0992-7158 (imprimé)

1718-8598 (numérique)

Découvrir la revue

Citer cet article

Yatribi, A. \& Nejmeddine, A. (2000). Impact écotoxicologique du traitement chimique des eaux usées de tanneries: Analyse technico-économique. Revue des sciences de l'eau / Journal of Water Science, 13(2), 107-118.

https://doi.org/10.7202/705384ar

\section{Résumé de l'article}

La toxicité des eaux usées du tannage au chrome et d'épilage-pelanage, avant et après traitement, a été évaluée par test Daphnia pulex. Cette évaluation a été déterminée à partir des équations de corrélation linéaires. Les résultats obtenus montrent que le tannage au chrome et l'épilage-pelanage présentent une toxicité élevée avec des valeurs des CI50 24h respectives de 0,15 et 3,36. Cette toxicité élevée pourrait être expliquée par la charge de ces effluents en chrome, en sulfures et en matière organique.

Après traitement par précipitation chimique, la CI50 24h a connu une importante augmentation. Elle est passée de 0,15 à 26,58 et de 3,36 à 11,1 respectivement pour le tannage au chrome et l'épilage-pelanage. Par conséquent, ces effluents traités peuvent être classés comme rejets peu toxiques. Cette diminution de la toxicité est liée surtout à l'abattement des MES (95\%), de la DCO (55\%), de Cr (90\%) et des sulfures (50\%). Ce traitement a donc, un double intérêt; le premier est environnemental, le second est économique du fait de la possibilité de recyclage du chrome récupéré dans le tannage du cuir. En effet, l'application expérimentale du chrome dans le tannage a montré un résultat similaire à celui obtenu par le produit commercial. Ainsi, suite à une analyse technico-économique, le bénéfice réalisé par cette opération est estimé à 3112,50 \$ par 1000 tonnes par an. 


\title{
Impact écotoxicologique du traitement chimique des eaux usées de tanneries : analyse technico- économique
}

\author{
Ecotoxicity impact of chemical treatment of tannery \\ wastewater: Technical-economic analysis
}

\section{A. YATRIBI, A. NEJMEDDINE *}

Reçu le 9 décembre 1999, accepté le 3 mars 2000**.

\section{SUMMARY}

\begin{abstract}
The purpose of our investigation was to evaluate the acute toxicity of chromium tanning and liming-deliming waste waters before and after chemical treatment. The invertebrate Daphnia pulex was used for toxicity test. The toxicity due to these industrial waste waters was revealed by the equations of the regression lines. The results of the toxicity test showed that the waste waters from chromium tanning and from de-hairingpresented higher toxicity, with respective $24-\mathrm{h}$ IC50 values of 0.15 and 3.36. This toxicity could be explained by higher levels of chromium, sulphides and organic matter in these waste waters.

After processing by chemical precipitation, 24-h IC50 values increased from $\mathbf{0 . 1 5}$ to $\mathbf{2 6 . 6}$ and from $\mathbf{3 . 4}$ to 11.1 respectively for the chromium tanning and the de-hairing waste waters. Consequently, these processed effluents can be classified as low toxic wastes. This diminution of the toxicity is linked to a reduction of suspended matter $(95 \%), \mathrm{COD}(55 \%), \mathrm{Cr}(90 \%)$ and sulphides $(50 \%)$. Hence, this treatment presents at least two advantages. The first is environmental, as just mentioned; the second is economic, since chromium can be recycled for reuse in leather tanning. The results suggest that the recovered chromium is similar to commercial tanning chromium. In fact, a techno-economical analysis showed that proposed treatment project can be economically beneficial (3112,5 dollars per 1000 tons per year).
\end{abstract}

Key-words: tannery, chromium, toxicity, chemical treatment, reuse, financial analysis, test daphnia.

Laboratoire d'écotoxicologie, Département de biologie, Université Cadi-Ayyad, Faculté des sciences Semlalia, BP 2390, Marrakech, Maroc

"Ce travail a été soutenu par l'Agence francophone de l'Enseignement supérieur et la recherche (AUPELF.UREF) dans le cadre d'une Allocation de recherche du fonds francophone de la recherche ". 


\section{RÉSUMÉ}

La toxicité des eaux usées du tannage au chrome et d'épilage-pelanage, avant et après traitement, a été évaluée par test Daphnia pulex. Cette évaluation a été déterminée à partir des équations de corrélation linéaires. Les résultats obtenus montrent que le tannage au chrome et l'épilage-pelanage présentent une toxicité élevée avec des valeurs des CI50 $24 \mathrm{~h}$ respectives de 0,15 et 3,36. Cette toxicité élevée pourrait être expliquée par la charge de ces effluents en chrome, en sulfures et en matière organique.

Après traitement par précipitation chimique, la CI50 $24 \mathrm{~h}$ a connu une importante augmentation. Elle est passée de 0,15 à 26,58 et de 3,36 à 11,1 respectivement pour le tannage au chrome et l'épilage-pelanage. Par conséquent, ces effluents traités peuvent être classés comme rejets peu toxiques. Cette diminution de la toxicité est liée surtout à l'abattement des MES (95\%), de la DCO (55\%), de Cr (90\%) et des sulfures (50 \%). Ce traitement a donc, un double intérêt ; le premier est environnemental, le second est économique du fait de la possibilité de recyclage du chrome récupéré dans le tannage du cuir. En effet, l'application expérimentale du chrome dans le tannage a montré un résultat similaire à celui obtenu par le produit commercial. Ainsi, suite à une analyse technico-économique, le bénéfice réalisé par cette opération est estimé à 3 112,5 dollars par 1000 tonnes par an.

Mots clés : tannerie, chrome, toxicité, traitement chimique, réutilisation, analyse financière, test Daphnie.

\section{1 - INTRODUCTION}

Le monde industriel est de plus en plus confronté au problème de contrôle des émissions de substances toxiques dans l'environnement, particulièrement sous forme d'effluent liquide (FÖRSTNER et WITTMANN, 1983). La complexité et la difficulté du problème résident dans la diversité des sources de rejets, dans l'importance quantitative de ces rejets et dans leur composition très variée (CRINE, 1993).

Au Maroc, à l'instar d'autres régions du pays, la ville de Marrakech connaît de multiples agressions quotidiennes engendrées par la pollution industrielle, en particulier celle des tanneries. Les eaux usées de la tannerie sont rejetées directement dans les milieux récepteurs (cas de l'Oued Issil) sans aucun traitement préalable.

II est admis que la présence accidentelle ou chronique de métaux lourds dans les déchets industriels (liquide et solide) peut atténuer gravement les capacités épuratrices des stations d'épuration, la qualité des eaux de surface et celle des eaux souterraines.

Les données relevées dans la littérature apparaissent, cependant, contradictoires quant à la toxicité et le devenir du $\mathrm{Cr}^{3+}$ dans les milieux récepteurs (SEMSARI et GAID, 1994). Les problèmes liés à la nuisance et à la tolérance du chrome sont assez complexes, en raison notamment, des phénomènes de précipitation et d'adsorption qui jouent certainement un rôle d'atténuation de la toxicité de cet élément (CHENG et al., 1975 ; NELSON et al., 1981). Afin de mettre en évidence le danger qui peut être engendré par les effluents de tannerie dans 
les milieux récepteurs et d'étudier l'impact écotoxicologique et environnemental du traitement chimique de ces effluents, nous nous sommes attachés à déterminer, avant et après traitement, la capacité inhibitrice des deux rejets les plus pollués du synoptique de fabrication du cuir à savoir l'épilage-pelanage et le tannage au chrome.

\section{2 - MATÉRIEL ET MÉTHODES}

\section{$2.1 \quad$ Réactifs}

Les caractéristiques des eaux usées brutes d'épilage-pelanage et du tannage au chrome étudiées sont présentées dans le tableau 1.

Tableau 1 Caractéristiques physicochimiques des eaux usées d'épilage-pelanage et du tannage au chrome.

Table 1 Physico-chemical characteristics of de-hairing and chromium tanning waste waters.

\begin{tabular}{|l|c|c|c|c|c|c|c|c|}
\hline & $\mathbf{p H}$ & $\begin{array}{c}\mathbf{D C O} \\
\left(\mathbf{m g} \cdot \mathbf{L}^{-1}\right)\end{array}$ & $\begin{array}{c}\mathbf{D B O}_{\mathbf{5}} \\
\left(\mathbf{m g} \cdot \mathbf{L}^{-1}\right)\end{array}$ & $\begin{array}{c}\mathbf{C l}^{-} \\
\left(\mathbf{m g} \cdot \mathbf{L}^{-1}\right)\end{array}$ & $\begin{array}{c}\text { Sulfures } \\
\left(\mathbf{m g} \cdot \mathbf{L}^{-1}\right)\end{array}$ & $\begin{array}{c}\mathbf{N H}^{4+} \\
\left(\mathbf{m g} \cdot \mathbf{L}^{-1}\right)\end{array}$ & $\begin{array}{c}\mathbf{C a}^{2+} \\
\left(\mathbf{m g} \cdot \mathbf{L}^{-1}\right)\end{array}$ & $\begin{array}{c}\mathbf{C r} \text { total } \\
\left(\mathbf{\mu g} \cdot \mathbf{L}^{-1}\right)\end{array}$ \\
\hline $\begin{array}{l}\text { Épilage- } \\
\text { pelanage }\end{array}$ & 12 & 20000 & 7000 & 160 & 2150 & 510 & 6900 & - \\
$\begin{array}{l}\text { Tannage } \\
\text { au chrome }\end{array}$ & $3-5,6$ & 7020 & 2400 & 2600 & 85 & 890 & 0,61 & 70000 \\
\hline
\end{tabular}

Toutes les dilutions ont été préparées par une eau minérale " eau Sidi-Ali " qui présentent les caractéristiques regroupées dans le tableau 2.

Tableau 2 Composition minérale de l'eau de «Sidi-Ali ».

Table 2 Mineral composition of "Sidi-Ali » water.

\begin{tabular}{|c|c|c|c|}
\hline Cations & $\begin{array}{c}\text { Concentration } \\
\left(\mathbf{m g} \cdot \mathbf{L}^{-1}\right)\end{array}$ & Anions & $\begin{array}{c}\text { Concentration } \\
\left(\mathbf{m g} \cdot \mathbf{L}^{-1}\right)\end{array}$ \\
\hline $\mathrm{Ca}^{2+}$ & 22,4 & $\mathrm{HCO}_{3}^{-}$ & 97,6 \\
$\mathrm{Mg}^{2+}$ & 7,3 & $\mathrm{Cl}^{-}$ & 18,5 \\
$\mathrm{Na}^{+}$ & 26,8 & $\mathrm{NO}_{3}^{-}$ & 5,4 \\
$\mathrm{~K}^{+}$ & 3,4 & $\mathrm{SO}_{4}^{2^{-}}$ & 31,6 \\
\hline
\end{tabular}

Tous les produits chimiques utilisés sont analytiquement purs (Merck). 


\subsection{Matériel biologique}

Le cladocère Daphnia pulex (LEYDIG, 1860) a été utilisé comme matériel biologique.

\subsection{Conditions d'élevage}

La souche de Daphnia pulex a été récoltée dans le lac réservoir Lalla Takerkoust (Maroc). Une seule femelle parthénogénétique a été maintenue en élevage dans l'eau minérale additionnée d'une culture d'algue Scenedesmus sp., dans un aquarium thermostaté à une température de $20 \pm 2{ }^{\circ} \mathrm{C}$. Les jeunes Daphnies, âgées de moins de $72 \mathrm{~h}$ et issues de la troisième génération, sont sélectionnées, maintenues à jeun pendant $24 \mathrm{~h}$ et utilisées comme matériel biologique dans ces tests d'immobilisation.

\subsection{Contrôle de la sensibilité}

La sensibilité du matériel biologique est déterminée à l'aide d'un test au bichromate de potassium mené parallèlement aux essais.

\subsection{Mode opératoire}

Le test d'immobilisation est réalisé selon la norme Afnor T90-301 (1983).

Les Daphnies sélectionnées sont mises dans des séries de tubes à essai en verre correspondant chacune à une dilution de l'effluent à tester. À chaque dilution correspond quatre répétitions. Chaque tube à essai reçoit cinq Daphnies et un volume final de $10 \mathrm{ml}$. Les tubes sont ensuite placés à l'obscurité dans des aquariums thermostatés à $20 \pm 2{ }^{\circ} \mathrm{C}$. Enfin, la mobilité des crustacés est déterminée, à $24 \mathrm{~h}$ et à $48 \mathrm{~h}$, par le dénombrement des Daphnies dans chaque tube après 15 secondes qui suivent une légère agitation du tube à essai. Cette opération nous a permis de représenter le pourcentage d'immobilisation en fonction de la concentration de l'effluent exprimée en pourcentage et d'en déduire la Cl50 $24 \mathrm{~h}$ et la $\mathrm{Cl} 5048 \mathrm{~h}$.

II est à noter que ces essais sont réalisés en deux étapes :

- l'essai préliminaire qui permet de déterminer l'intervalle de dilutions de l'effluent qui fait varier le pourcentage d'immobilisation de $0 \%$ à $100 \%$;

- l'essai définitif qui permet de déterminer les pourcentages des Daphnies immobilisées en $24 \mathrm{~h}$ et en $48 \mathrm{~h}$ par les différentes concentrations examinées et d'en déduire la $\mathrm{Cl} 5024 \mathrm{~h}$ et la $\mathrm{Cl} 5048 \mathrm{~h}$.

\subsection{Validité des résultats}

Enfin, pour confirmer la validité de nos résultats, nous notons à l'issu de chaque test la concentration finale en oxygène dissous et le $\mathrm{pH}$ de chaque solution. Un test au bichromate de potassium est aussi réalisé afin de vérifier l'homogénéité et la sensibilité du lot des crustacés utilisés.

\subsection{Traitement des effluents étudiés}

\subsubsection{Effluent du tannage au chrome}

Généralement, le traitement des effluents liquides par précipitation consiste en la transformation de composés métalliques fortement dissociés (sels en 
solution) en composés très peu solubles qui peuvent être récupérés par des techniques classiques de séparation liquide-solide tels que la filtration, la sédimentation ou la flottation (CRINE, 1993). Cette opération implique toujours l'ajout d'un réactif de précipitation réalisant la transformation souhaitée (CUSHINE, 1984). Les développements les plus récents tendent à améliorer les performances de ce procédé en terme d'épuration, c'est-à-dire de façon à atteindre des concentrations résiduelles de plus en plus faibles. Les limites sont liées d'une part à la solubilité des composés métalliques formés et, d'autres part, aux possibilités de récupération de ces composés ainsi formés (CRINE, 1993).

Le traitement effectué, concernant les eaux usées du tannage au chrome, consiste en une précipitation à différents $\mathrm{pH}$ par le carbonate de sodium $\left(\mathrm{Na}_{2} \mathrm{CO}_{3}\right)$.

Le dosage du chrome a été effectué par absorption atomique équipée d'un four à graphite (type Varian AA 20).

\subsubsection{Effluent d'épilage-pelanage}

Le traitement de l'effluent d'épilage-pelanage consiste en une élimination et récupération des composés sulfurés sans procéder à leur dégradation.

Dans un bêcher de $250 \mathrm{~mL}$, un volume de $200 \mathrm{~mL}$ d'eau usée d'épilagepelanage est additionné à $20 \mathrm{~mL}$ d'une solution de sulfates ferreux, neutralisé par $\mathrm{NaOH}$ puis laissé précipiter pendant $4 \mathrm{~h}$. Ensuite, les deux phases sont séparées mécaniquement et seule la phase liquide a été analysée.

\section{3 - RÉSULTATS ET DISCUSSION}

\subsection{Détermination des concentrations inhibitrices moyennes (Cl50)}

Après $24 \mathrm{~h}$ et $48 \mathrm{~h}$, nous avons déterminé les concentrations moyennes inhibitrices : $\mathrm{Cl} 5024 \mathrm{~h}$ et $\mathrm{Cl} 5048 \mathrm{~h}$, qui provoquent l'immobilisation de $50 \%$ d'une population de Daphnies testées. Les résultats concernent en premier lieu les eaux usées d'épilage-pelanage et celles du tannage au chrome non traitées (tableau 3).

Tableau 3 Détermination de la Cl50 24 h et 48 h sur Daphnia pulex de l'effluent d'épilage- pelanage et du tannage au chrome brut.

Table 3 Determination of 24- $h$ and 48-h IC50 values for raw de-hairing and chromium tanning effluents.

\begin{tabular}{|l|c|c|c|c|}
\hline & $\begin{array}{c}\text { CI50 24 h } \\
\text { en \% de l'effluent }\end{array}$ & $\begin{array}{c}\text { CI50 48 h } \\
\text { en \% de l'effluent }\end{array}$ & $\begin{array}{c}\mathbf{0}_{\mathbf{2}} \text { dissous } \\
\mathbf{m g} \cdot \mathbf{L}^{-1}\end{array}$ & pH \\
\hline Épilage-pelanage brut & 3,36 & 2,80 & 5,4 & 8,2 \\
\hline Tannage Cr. brut & 0,15 & 0,01 & 5,3 & 7,1 \\
\hline
\end{tabular}




\subsection{Validité des résultats}

Les résultats que nous avons obtenu (tableau 3) sont considérés valables puisqu'ils vérifient les trois conditions suivantes:

1) La teneur en oxygène dissous mesurée en fin d'essai est supérieure à $2 \mathrm{mg} \cdot \mathrm{L}^{-1}$.

2) Le pourcentage d'immobilisation observé dans les tubes témoins (2\%) est inférieur à $10 \%$.

3) La Cl50 $24 \mathrm{~h}$ du bichromate de potassium est égale à $1,1 \mathrm{mg} \cdot \mathrm{L}^{-1}$, elle est donc comprise entre 0,9 et $1,5 \mathrm{mg} \cdot \mathrm{L}^{-1}$.

\subsection{Discussion}

Les résultats figurant dans le tableau 3 montrent que:

- les Cl50 $24 \mathrm{~h}$ et Cl50 $48 \mathrm{~h}$ du tannage au $\mathrm{Cr}$ non traité sont largement inférieures (au moins 22 fois) à celles d'épilage-pelanage. La richesse des eaux usées d'épilage-pelanage en matière organique peut être à l'origine de cette différence de toxicité entre ces deux effluents. En effet, d'après JORGENSEN (1976), la possibilité d'utilisation de la matière organique dissoute par les organismes aquatiques a été beaucoup discutée. SALONEN et HAMMAR (1986) ont montré que la matière organique joue un rôle majeur dans la nutrition des invertébrés. Généralement, la toxicité de ces deux rejets est donc le résultat d'un effet combiné des substances organiques et minérales contenues dans chaque effluent. En réalité, il est difficile de corréler les valeurs des $\mathrm{CI} 5024 \mathrm{~h}$ à celles des paramètres physicochimiques vu que les eaux usées de ces effluents testés sont des matrices très complexes. En revanche, on peut remarquer qu'il y a une différence notable entre ces deux effluents. L'effluent d'épilage-pelanage est surtout riche en matière organique ( $\mathrm{DCO}=20000 \mathrm{mg} \cdot \mathrm{L}^{-1}$ ); alors que le second est riche en chrome et en matière organique. Ces micropolluants sont doués d'une forte toxicité envers les organismes aquatiques. En effet, OIKARI et al. (1992) ont montré que le chrome présente une toxicité très élevée vis-à-vis du Daphnia magna ;

- la concentration en chrome total correspondant à la CI50 $24 \mathrm{~h}\left(105 \mu \mathrm{g} \cdot \mathrm{L}^{-1}\right)$ du tannage au chrome brut est inférieure à celle de $\mathrm{K}_{2} \mathrm{Cr}_{2} \mathrm{O}_{7}(290 \mu \mathrm{g}$ $\mathrm{Cr}^{6+} \cdot \mathrm{L}^{-1}$ ). Ce qui affirme que la toxicité des eaux usées du tannage au chrome brut est due non seulement au chrome, mais à d'autres produits chimiques utilisés lors du processus de fabrication comme les ammoniums, les sulfures, etc.

\subsection{Influence du traitement sur la CI50}

\subsubsection{Résultats des analyses physicochimiques après traitement}

L'eau usée du tannage au chrome est ajustée à différents $\mathrm{pH}$ par ajout de $\mathrm{Na}_{2} \mathrm{CO}_{3}$. Pour chaque $\mathrm{pH}$, nous avons réalisé des analyses dont les résultats sont représentés par les figures 1, 2 et 3 .

À partir de la figure 1, on constate que les MES évoluent d'une manière décroissante en fonction $\mathrm{du} \mathrm{pH}$. Cela peut être expliqué par une sédimentation 
naturelle des grosses particules et par la précipitation des éléments métalliques et organiques dissous et particulaires. On remarque aussi qu'à partir d'un $\mathrm{pH}=9$, le pourcentage d'abattement reste constant et maximal et atteint $95 \%$.

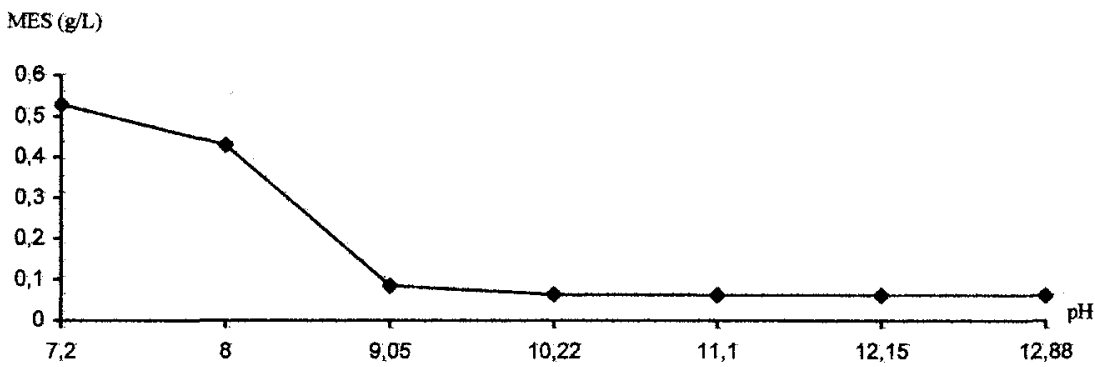

Figure 1 Évolution des matières en suspension en fonction du pH.

Suspended solid evolution vs. $\mathrm{pH}$.

L'évolution des teneurs en chrome en fonction du pH (figure 2) est semblable à celle des MES. Cette courbe montre que plus le $\mathrm{pH}$ augmente, plus la précipitation de $\mathrm{Cr}$ est favorisée. Aux $\mathrm{pH}$ très élevés, nous avons constaté une légère re-solubilisation de $\mathrm{Cr}$. En effet, à ces $\mathrm{pH}$, les hydroxydes métalliques se comportent comme des acides et se dissocient en ions. À $\mathrm{pH}=9,4$, la précipitation est maximale et le $\mathrm{Cr}$ récupéré est évalué à $95 \%$ de $\mathrm{Cr}$ total en solution.

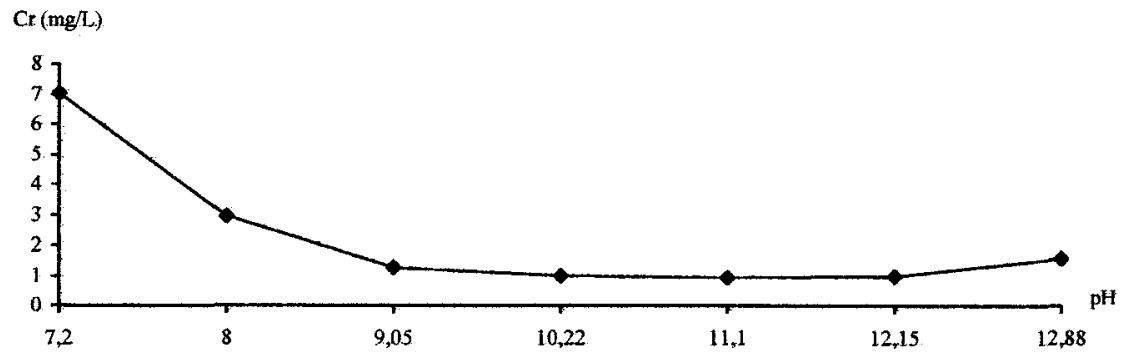

Figure 2 Évolution des teneurs en chrome en fonction du $\mathrm{pH}$.

Chromium levels evolution vs. $\mathrm{pH}$.

La courbe de la DCO en fonction du pH (figure 3) montre que le pourcentage maximal d'abattement de la matière organique (55\%) est obtenu à $\mathrm{pH}=10$.

Le traitement a permis une élimination de $95 \%$ des matières en suspension, de $55 \%$ de la DCO et d'environ $90 \%$ du chrome qui peut être recyclé dans l'industrie elle même.

Quant à l'effluent d'épilage-pelanage, le dosage des sulfures après décantation, montre que le rendement du traitement proposé est évalué à $54 \%$.

Le résultat que nous avons trouvé est comparable à celui trouvé par COLLIVIGNARELLI et BARDUCCi (1984). Ces résultats peuvent aussi s'améliorer en procédant à une filtration sous vide au lieu d'une décantation. 


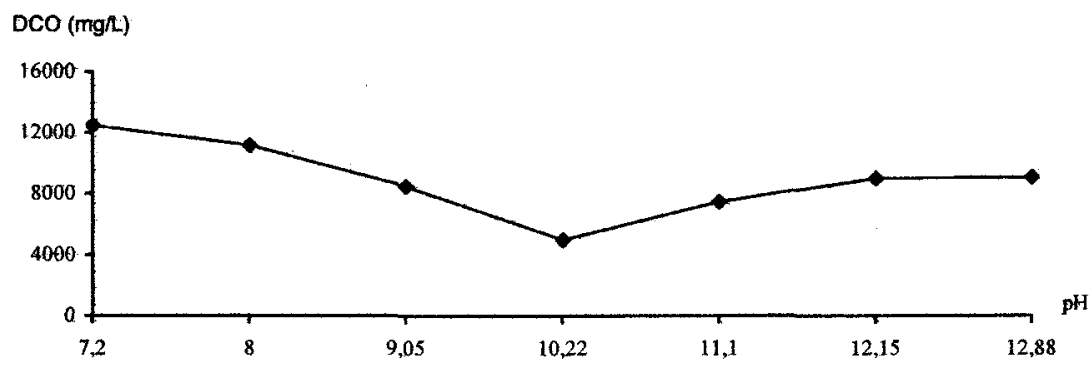

Figure 3 Évolution de la DCO en fonction du $\mathrm{pH}$.

COD evolution vs. $\mathrm{pH}$.

\subsubsection{Bilan économique du traitement}

La récupération de la totalité des eaux résiduaires chromées pour la régénération du chrome est une opération faisable mais elle est limitée par la mise en place d'investissement qui reste envisageable plus au niveau collectif qu'au niveau individuel. Pour avoir une idée sur l'économie lié au procédé de traitement et de récupération du chrome, nous exposons l'expérience effectuée dans une tannerie qui se résume ainsi :

\section{Présentation de l'usine}

L'usine traite 1000 t/an de peaux de veaux destinées à la chaussure et à la maroquinerie.

\section{Bilans avant travaux}

* $36 \mathrm{~kg}$ de $\mathrm{Cr}_{2} \mathrm{O}_{3}$ consommé par tonne de peaux en tripe (36 t/an).

\section{Objectifs}

L'objectif consiste à récupérer le chrome pour permettre l'utilisation des boues de la station d'épuration dans le domaine agricole et le recyclage de ce métal dans le circuit de fabrication du cuir pour économiser sur le produit neuf.

La précipitation de $1 \mathrm{~kg}$ de $\mathrm{Cr}_{2} \mathrm{O}_{3}$ nécessite $1,63 \mathrm{~kg}$ de soude tandis que la redissolution demande 1,3 litres de $\mathrm{H}_{2} \mathrm{SO}_{4}$.

\section{Bilan économique}

Les charges globales ont été évaluées à $306375 \mathrm{DH}(1 \mathrm{US} \$=10 \mathrm{DH})$ et qui peuvent être réparties comme suit:

Réactifs

$82875 \mathrm{DH}$

Main d'œuvre

Énergie d'entretien

$73500 \mathrm{DH}$

Contrôles

Amortissement 


\section{Gains}

Le coût de $\mathrm{Cr}_{2} \mathrm{O}_{3}$ récupéré $\left(11,2\right.$ tonnes à $\left.30 \mathrm{DH} \cdot \mathrm{kg}^{-1}\right)$ est évalué à $337500 \mathrm{DH}$. Par conséquent, le gain est de l'ordre de $31125 \mathrm{DH}$ par an.

\section{Analyse financière du projet}

Plusieurs méthodes sont utilisées dans l'évaluation de la rentabilité financière des projets d'investissement. Les projets de prévention de la pollution peuvent et doivent être soumis aux mêmes règles d'évaluation. Parmi les méthodes d'analyse financière les plus utilisées, figure le temps de retour brut (ou temps de remboursement simplifié). II s'agit de la mesure la plus simple de la rentabilité financière d'un projet. Ce temps mesure la période nécessaire pour récupérer, à travers les bénéfices réalisés, le capital investi dans le projet. Dans ce cas où le bénéfice annuel est constant, le temps de retour brut est donné par la formule :

$$
\text { Temps de retour brut (ans) }=\frac{\text { coût total de projet }}{\text { bénéfice annuel }}
$$

II faut signaler que ce temps de retour brut ne tient pas compte des impôts. Dans le cas contraire, il faut faire appel à une autre grandeur d'analyse similaire à la notion du temps de retour brut qui est le taux de retour sur l'investissement défini par:

$$
\text { Taux de retour }(\%)=\frac{\text { bénéfice annuel net }}{\text { coût total du projet }}
$$

Le temps de retour brut calculé pour ce projet est d'environ 10 ans. C'est un temps relativement élevé, mais il faut que ce facteur présente quelques inconvénients pouvant entraîner une comparaison erronée des projets. En particulier, cette méthode ne tient pas compte des bénéfices réalisées après le point mort.

Il faut signaler que même si le temps de retour brut est de 10 ans, ce projet aura un autre intérêt d'ordre environnemental du fait de la réduction de la pollution des eaux du tannage au chrome.

\subsection{Réutilisation du chrome récupéré dans le tannage du cuir}

L'objectif de cette expérience est d'évaluer le pouvoir tannant du produit récupéré et de le comparer avec le produit commercial utilisé en industrie. La re-solubilisation du chrome est réalisée à $\mathrm{pH}=3$ par ajout de $\mathrm{H}_{2} \mathrm{SO}_{4}$ selon la réaction chimique suivante:

$$
2 \mathrm{Cr}(\mathrm{OH})_{3}, 3 \mathrm{H}_{2} \mathrm{O}+3 \mathrm{H}_{2} \mathrm{SO}_{4} \rightleftarrows 2 \mathrm{Cr}^{3^{+}}+3 \mathrm{SO}_{4}^{2^{-}}+3 \mathrm{H}_{2} \mathrm{O}
$$

La solution ainsi obtenue est prête pour le tannage. En effet, une prise d'essai de $100 \mathrm{~g}$ de peaux (Bovin et Ovin) est soumise en utilisant le Cr recyclé et le $(8 \mathrm{~g})$ et le $\mathrm{Cr}$ commercial $(8 \mathrm{~g})$.

Après le tannage, nous avons dosé la quantité de Cr fixé par la peau. Les résultats sont exprimés en pourcentage massique du chrome (tableau 4). 
Tableau 4 Pourcentage du chrome absorbé par $100 \mathrm{~g}$ de peaux tannées à $\mathrm{pH}=3,8$.

Table 4 Percent chromium absorbed by $100 \mathrm{~g}$ of tanned leather at $\mathrm{pH}=3.8$.

\begin{tabular}{|l|c|c|}
\hline & Cr. recyclé & Cr. commercial \\
\hline Peau de bovin & $2,30 \%$ & $2,31 \%$ \\
Peau d'ovin & $2,30 \%$ & $2,30 \%$ \\
\hline
\end{tabular}

Les résultats obtenus, montrent que le tannage réalisé par le chrome récupéré est similaire à celui effectué par le produit commercial. En effet, la quantité du chrome fixé et la quantité du cuir tanné sont semblables pour les deux produits utilisés. Ces résultats montrent aussi que la fixation du chrome ne dépend pas de la nature de la peau testée.

\subsection{Impact du traitement sur la toxicité}

\subsubsection{Détermination de la CI50}

Les résultats présentés dans le tableau 5 , relatifs à l'eau récupérée après un traitement à un $\mathrm{pH}$ de 9,4 , montrent que :

- les Cl50 24 h et Cl50 48 h de l'effluent du tannage au chrome brut ont augmenté après le traitement. Elles passent respectivement de 0,15 et 0,01 à 26,57 et 17,76. De même, celles de l'effluent d'épilage-pelanage ont passé respectivement de 3,36 et 2,8 à 11,1 et 9,88 . Par conséquent, la toxicité de cette eau usée traitée a diminué. Cette diminution peut être liée au pourcentage d'abattement des MES, de la DCO et du Cr. En effet, la toxicité est le résultat d'un effet combiné d'une multitude de polluants chimiques minéraux et organiques biodisponibles et de leurs produits de dégradation (MAÂROUF et al., 1994). Les interactions synergiques, antagonistes et/ou additives de ces micropolluants sont très complexes et difficiles à prévoir (HOCKETT et MOUNT, 1996 ; LALANDE et PINEL-ALLOUL, 1986) ;

- le classement par ordre croissant du pouvoir inhibiteur des quatre rejets étudiés par rapport à la Cl50 $24 \mathrm{~h}$ est le suivant : Tannage au Cr brut $>>$ Épilage-pelanage brut > Épilage-pelanage traité > Tannage au Cr traité.

Tableau 5 Classement des quatre rejets étudiés par ordre de toxicité.

Table 5 Classification of the four studied effluents in order of their toxicity.

\begin{tabular}{|l|c|c|c|c|}
\hline Effluent & $\begin{array}{c}\text { Épilage-pelanage } \\
\text { brut }\end{array}$ & $\begin{array}{c}\text { Épilage-pelanage } \\
\text { traité }\end{array}$ & $\begin{array}{c}\text { Tannage } \\
\text { brut }\end{array}$ & $\begin{array}{c}\text { Tannage } \\
\text { traité }\end{array}$ \\
\hline Cl50 $24 \mathrm{~h}$ & 3,36 & 11,1 & 0,15 & 26,57 \\
CI50 $48 \mathrm{~h}$ & 3,36 & 9,88 & 0,15 & 17,76 \\
UT $(\%)$ & $10<29,7<100$ & $1<9<10$ & $673>100$ & $1<3,7<10$ \\
Degré de toxicité & Toxique & Peu Toxique & Très toxique & Peu toxique \\
\hline
\end{tabular}


En 1986, VASSEUR et al. ont classé les rejets industriels par ordre de toxicité en fonction des valeurs de leurs $\mathrm{Cl} 5024 \mathrm{~h}$ exprimées en pourcentage d'effluent ou en unité toxique (UT $=1 \times 100 / \mathrm{Cl} 5024 \mathrm{~h})$. Les résultats sont rassemblés dans le tableau 5.

Selon cette classification, le test Daphnie permet donc, de classer le rejet du tannage au chrome brut comme étant très toxique, l'effluent d'épilage-pelanage brut comme étant toxique et ceux du tannage au chrome et d'épilagepelanage traités comme peu toxiques.

La comparaison des résultats est très difficile vu que les conditions des tests et les caractéristiques physicochimiques et métalliques des effluents varient énormément en fonction du temps, sans oublier la composition physicochimique des milieux de dilution utilisés par les normes OCDE (1984) (eau douce) et les normes ISO (1983) (eau synthétique). II est donc nécessaire de faire varier les tests pour bien cerner le degré de toxicité d'un effluent vu que leurs effets se manifestent différemment à différents niveaux trophiques.

\section{4 - CONCLUSION}

Au cours de cette étude, nous avons pu mettre en évidence la toxicité de quatre effluents (Épilage-pelange et Tannage au chrome bruts et traités) moyennant le test «Daphnie » (24 h et $48 \mathrm{~h}$ ). Les résultats obtenus ont permis de classer ces quatre effluents, en se basant sur la Cl50 $24 \mathrm{~h}$, comme suit :

Tannage au Cr brut > Épilage-pelanage brut >> Épilage-pelanage traité $>$ Tannage au Cr traité.

Nous avons constaté aussi, que la $\mathrm{Cl} 5024$ h est toujours supérieure à la $\mathrm{Cl} 50$ $48 \mathrm{~h}$. En effet, la durée d'exposition de la daphnie à la substance toxique est un facteur important dans la mesure où il fait apparaître une différence remarquable entre les CI50 $24 \mathrm{~h}$ et $48 \mathrm{~h}$ à la température d'expérimentation (BELABED et al., 1994). Ces mêmes auteurs ont montré que le test «Daphnie » dépend aussi de la température. En effet, une simple augmentation de la température favorise la solubilité des métaux et la désoxygénation des milieux d'essai.

L'évaluation de la toxicité de ces effluents industriels, dont l'intérêt est d'ordre pratique, permet de prévoir les précautions à prendre avant de rejeter les effluents à l'état brut.

L'élimination de la matière organique, des matières en suspension, du chrome et des sulfures a diminué la toxicité des deux effluents en les faisant passer d'un rejet très toxique à un rejet peu toxique. Ce gain sur le plan environnemental est accompagné d'un second d'ordre économique justifié par le recyclage du chrome au sein de l'industrie. En effet les résultats obtenus montrent que le tannage effectué par le chrome récupéré après traitement chimique est similaire à celui effectué par le produit commercial.

Le revenu économique du recyclage du chrome après traitement de 1000 tonnes de peaux est évalué à $31125 \mathrm{DH} / \mathrm{an}$. Le temps de retour brut est de 10 ans. C'est un temps relativement élevé, mais le projet reste d'un intérêt 
considérable surtout d'ordre environnemental du fait de la réduction de la pollution des eaux usées du tannage au chrome.

\section{RÉFÉRENCES BIBLIOGRAPHIQUES}

AFNOR, 1983. Essai des eaux. Détermination de l'inhibition de la mobilité de Daphnie magna. Norme expérimentale homologuée T90-301, 1-11.

BELABED W., KESTALI N., SEMSARI S., GAID A., 1994. Évaluation de la toxicité de quelques métaux lourds à l'aide du test Daphnie. Techniques Sciences et Méthodes, 6.

CHENG M.H., PATTERSON J.W., MINEAR R.A., 1975. Heavy metals uptake by activated sludge. J. Water Pollut. Control Fed., 47, 362-370.

CRINE M., 1993. Le traitement des eaux usées industrielles chargées en métaux lourds. Situation actuelle et perspectives de développement. Tribune de l'eau, $561 / 1$, janvier-février.

CUSHINE G., 1984. Removal of metals from wastewater : neutralization and precipitation, Pollution Technology Review, 107, Noyes Data Corp., Park Ridge, N.J.

FÖRSTNER U., WITTMANN G.T.W., 1983. Metal pollution in the environment, Springer Verlag, Berlin.

HOCKETT L., MOUNT K., 1996. Use of metal chelating agents to differentiate among sources of aquatic toxicity. Environ. Toxicol. Chem., 15, 1687-1693.

ISO, 1983. Toxicité à long terme vis-à-vis des Daphnies, résultats de l'enquête « Essai de reproduction ", ISO/TC 147/SC. 5 GT. 2N. $17 \mathrm{p}$.

LALANDE M., PINEL-ALLOUL B., 1986. Acute toxicity of cadmium, copper and zinc to Topocyclops prasinus mexicanus (Cyclopoida, Copepoda) from three Quebec lakes. Environ. Toxicol. Chem., 5, 95-102.
JORGENSEN C.B., 1976. August Pütter, Augst Krogh and modern ideas on the use of dissolved organic matter in aquatic environments. Biol. Rev., 51, 291-328.

MAÂROUF M., MAZLANI S., PIHAN J.C., BELKHADIR M., 1994. Comparaison de la sensibilité de deux crustacés : Gammarus gauthieri et Daphnia magna, à la toxicité létale des extraits de sédiments de quatre cours d'eau marocains. Ann/s Limnol, 30 (3), 197-207.

NELSON P.O., CHUNG A.K., HUDSON M.C, 1981. Factors affecting the fate of heavy metals in the activated sludge process. J. Water Poll. Control Fed., 53, 13231332.

OCDE, 1984. Daphnia sp. Essai d'immobilisation immédiate et essai de reproduction sur 14 jours. Ligne directive de l'OCDE pour les essais de produits chimiques, 202, $17 p$.

OIKARI, KUKKONEN, VIRTANEN, 1992. Acute toxicity of chemicals to Daphnia magna in humic waters. Sci. Tot. Environm., 117-118, 367-377.

SALONEN K., HAMMAR T., 1986. On the importance of dissolved organic matter in the nutrition of zooplancton in some lake waters. Oecologia (Berlin) 68, 246-253.

SEMSARI S., GAID A., 1994. Inhibition of chromium (III) on the activated sludge activity. Environm. Technol, , 15, 255-262.

VASSEUR P., BOIS F., FÉRARD J.F., RAST C., LARBAIGT G., 1986. Influence of physico-chemical parameters on the Microtox Test Response. Toxicity Assessment: An International Quarterly, Report 1, 283-300. 